\title{
Digitizing the Informed Consent: the challenges to design for practices
}

\author{
Michela Assale ${ }^{1,2}$, Erica Barbero ${ }^{1}$, and Federico Cabitza*1 \\ ${ }^{1}$ University of Milano-Bicocca, Milan, Italy \\ Email: federico.cabitzacunimib.it \\ ${ }^{2} \mathrm{~K}$-Tree srl, Milan, Italy
}

\begin{abstract}
This paper reports a user study performed to assess the usability of a Web-based electronic informed consent application called DICE, which is aimed at supporting patients in the process of reading, understanding and using the informed consent as a trigger for further interaction with the team of care givers. In particular, we performed a questionnaire-based study and a series of individual semi-structured interviews to understand whether the application is usable and can be used in real-world settings, respectively. We found that patients could appreciate the availability of interactive tools like DICE, but health professionals believe that its actual adoption in current workflows and practices could be hampered by the chronic lack of time and health operators who could timely address the licit requests that such a tool could bring to light.
\end{abstract}

Keywords-informed consent; usability; doctor-patient communication

\section{Motivations And BACKGROUnd}

The idea that physicians should get an informed consent by patients before they undergo a treatment has been part of modern medical practice since its inception: the discussion of what the "reasonable physician" should do to inform his patients appeared in 1767 [1] and the legal concept stabilised in the early 1900s for surgical cases, mainly to relieve surgeons from the charge of "unauthorised touching" and intentional harm. Since those days until the 1970s "what constituted an adequate medical informed consent was determined by the medical community" [1]. In the 1970 s an increasing number of courts in negligence cases convened that "what constituted adequate information for informed consent was what a "reasonable person" would want to know. Since then, little has changed and the concept of "reasonable person", as vague as it may sound, has been mirrored by a one-size-fits-all approach to informed consent. Therefore, one critical element that the digitisation of informed consent is called to address is how to reconcile this concept with the single patient idiosyncrasies, in regard to education, expectations, attitudes, fears and familiarity with medical concepts.

This paper focuses on a Web-based online application that we designed to address the peculiar needs of single patients in getting properly informed about the interventions they are to undergo. To this aim, we took the perspective that sees the Informed Consent (IC) not only as a stipulation, which ratifies the agreement between the patient and their doctors upon the procedures that the former one will undertake to solve a health problem, but also and above all as a process itself, a trajectory of conviction, in which the patient learns something about the medical procedures, and even themselves.

The processual element of the IC is part of a wider process of patient-doctor communication, in which the former ones express preferences, worries and expectations about the health problems they manifest; and the latter ones propose their best options to solve those problems, presenting the pros and cons of each of those options.

In the specialist literature, many studies have observed as, mainly due to the excessive length of informed consent forms, or the excessively technical or involute language expressed therein, the stipulation element (that is inevitable and intrinsic to any IC) is predominant with respect to the learning one, and in some cases this latter one is negligible [2]: this results in an IC that is seen just as an administrative task devoid of communication between patients and care givers, and of learning and awareness by the patients. Moreover, several studies have observed that many patients do not understand the document they are signing [2]-[4]. Consequently, ill-informed patients tend to either overestimate risks [5], thus leading to greater pre-operative anxiety, which has been found to correlate with worse recovery [6], as well as major morbidity and mortality [7]; or to overestimate prospects, which can result in higher rates of medical complaints [8] and subsequent distress, which pose different and equally relevant concerns from the ethical point of view [3].

In this paper, we will describe the mixed-methods research [9] that we undertook to assess the usability and adoptability of a Web application, called Digital Informed Consent Experience (DICE). We developed it to improve the patients' experience of the IC process and make it more tailored to their information and emotional needs. In Section II, we mention some work on electronic IC that inspired the design of DICE. In Section III, we briefly describe the DICE application. In Sections IV and V, we will report the main findings from the user studies where we administered both closed-ended psychometric questionnaires 
to a sample of prospective users, and an open-ended semistructured interview with some key domain experts. In Section VI, we discuss the findings from the above user studies and summarise their main contributions.

\section{RELATED WORK}

In March 2015, the US Food and Drug Administration (FDA) released a draft guide with recommendations for the implementation of electronic Informed Consent (eIC, or eConsent) applications ${ }^{1}$. In this guide, it is stated that "any eIC should be easy to navigate, allowing the user to proceed forward or backward within the system and to stop and continue at a later time". Nowadays, the use of eIC applications is widespread, especially in the context of clinical trial recruitment where the IC text is conveyed in a structured format and its electronic version is aimed at maximising the participants' understanding and engagement throughout the course of the study [10].

In the clinical trial domain, several comparative studies have been performed to compare paper-based and interactive electronic IC forms. For instance, in [11], the authors report that patients who used the electronic IC form got significantly higher comprehension scores than paper-based consent participants, while the difference was not significant in regard to overall satisfaction and enjoyment. In general, other characteristics impact on patient satisfaction and comprehension than merely the support format, like brevity [4], [12] and visual richness, which nevertheless can be significantly higher when the IC is conveyed through an electronic interactive application [11], [13].

Much less research has been so far accomplished on eIC in the domains of primary care and secondary (i.e. inhospital) care. This distinction is relevant as the IC task is different according to the setting and in regard to the typology of patients, the processes of which the consent is part, and its purpose. In primary care settings, the IC is mainly addressed to healthy (or not critically ill) people, and it is mainly related to the permission to manage personal and sensitive data by the general practitioners and their assistants. On the other hand, in secondary and clinical research settings, the IC is related to the purpose of privacy permission, as well as to inform the patients of the procedures that are proposed to them and the associated risks.

In the specialist literature, authors report many benefits for eIC applications in respect to their paper-based counterparts. In both [14] and [15], groups of researchers implemented an eIC: The results showed that the great majority of the surveyed patients preferred the digital IC compared to the traditional ones. Benefits also include operational improvements for research sponsors and clinical administrators [16]

\footnotetext{
${ }^{1}$ Use of Electronic Informed Consent in Clinical Investigations Questions and Answers Guidance for Industry Draft Guidance; March, 2015. https: //www.fda.gov/downloads/drugs/guidances/ucm436811.pdf. [Last accessed on 2018 Dec 19]
}

as data extracted from eIC are usually collected in a centralised repository, and this allows for data aggregation, realtime access and analysis at almost no additional cost.

Rowan et al. (2017) [17] mention a work by Hochhauser (2015) by enumerating a list of usability issues associated with eIC. These include: (1) reading from a device being more time consuming; (2) presenting too much information on multiple small screens; (3) not all users being technologically savvy; (4) reading from devices not allowing for deeper processing of information; and, (5) text not being legible on a device. Also Wilbanks (2018) [18] recently discussed pros and cons of eIC. Among the shortcomings, the author notices that people read and comprehend text on a computer screen more slowly than they read and understand printed material.

As hinted in Section I, many studies [19], [20] report that one of the main problems related to the filling of the IC forms is that the language used is not totally comprehensible to most people, and this creates an even worse divide with poor-literacy patients, who nevertheless should not be excluded from participation to either trial studies or standard care [21]. Although these and similar studies found that eIC applications can improve the patients' understanding, a systematic review [22] proposed talking to the patient one-on-one as the most effective way to improve the consent experience. This is true also considering that the consent experience cannot be traced back solely to the cognitive dimension (i.e. understanding and comprehension), which nevertheless is the main concern of most of literature contributions [13]. Studies usually focus on decision-relevant variables, like comprehension, risk/benefit awareness, and trust feeling [12], while the emotional dimension, which is related to how patients communicate worries and concerns and have these addressed by the care givers, is still neglected. This is why we conceived our system more as a trigger for patient-care giver interaction than as a stand-alone application aimed at improving the comprehension of a written document, that is as a simple component of a wider and more inter-personal management of both the cognitive and emotional needs of patients.

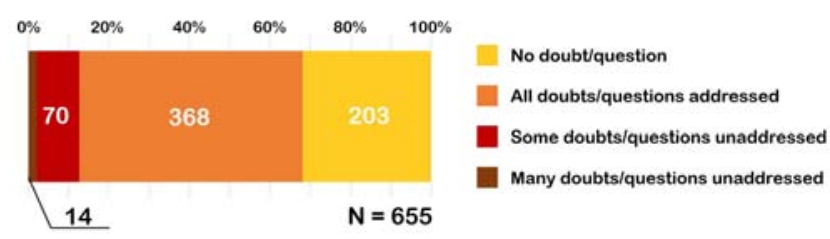

Figure 1. Response proportions from the preliminary patient survey. 


\section{DICE: MAIN FEATURES AND INTERFACE}

The DICE application is a web-based eIC application that can be accessed online ${ }^{2}$ via a standard Web browser. The main idea underlying DICE is to make the reading experience of the IC more interactive and tailored for the patients, as well as a trigger for more and better face-toface interactions between them and the caring staff. This is accomplished through the annotation of the IC text: on one hand, the system automatically annotates (i.e. enriches) the IC text with on-demand medical definitions. On the other, the patients can annotate those passages of the IC that either worry them or they don't understand, and request the interventions of doctors and nurses on those points.

We investigated the potential of this latter functionality quite early in the design of DICE. To this aim, we undertook a preliminary study at the IRCCS Orthopedic Institute Galeazzi, a large teaching hospital based in Milan (Italy) where almost 5,000 surgeries are performed yearly, mostly hip and knee prosthetic surgery and spine-related procedures. For 8 months (March 2017 - October 2018) we sent an email to a random sample of patients who had undertaken surgery at Galeazzi a few weeks earlier to invite them to fill in an online questionnaire and answer the following item: "When you signed the informed consent and approved the following treatment, did you feel the need to ask more questions or ask for more clarifications to your care givers than you actually did?". We collected the opinions of 701 patients; however, 46 of them admitted they could not remember the IC task. Therefore we got 655 complete answers (see Figure 1) to analyse. Only one third of the respondents claimed they had not had any question to ask in regard to the informed consent, while slightly more than half of the respondents claimed that all of their doubts and questions had been expressed and hence addressed adequately by the caring staff. Notably, yet, approximately $12 \%$ of participants claimed that they did have more questions to ask than those that they had actually asked (for any reason that we did not further investigate): indeed, one fourth of these respondents claimed they had many questions to ask (see Figure 1) and hence many points or doubts that had been left unaddressed. This meant for us that DICE, with its role in facilitating the request for help by patients, could directly support at least one patient out of two, to both focus on those passages of the IC that would require higher awareness and attention and, more notably, to help patients "raise their hands", get the caregivers' attention and ask for more clarifications or reassurances.

In what follows, we briefly describe the DICE interface. Users can get access to the text of any informed consent that has been uploaded after a regular login page (the current

\footnotetext{
${ }^{2}$ The code and a full-functional demo is available online on the Github platform at the following address: https://github.com/ericab12/ informed-consent-v2
}

version accepts documents in either simple text or HTML format). In the regular business case that we drew, health professionals either upload or select the informed consent that the inpatient has to read, log into the system and hand out a tablet to the patient with a DICE instance in foreground, awaiting for user input.

The main application screen is divided in 2 parts (see Figure 2): the top part, or header, is always visible and contains the title of the IC, and the command bar; the bottom and larger part contains the scrollable and multipage body of the IC text, divided in smaller paragraphs; each paragraph can be annotated by the patient by clicking on two specific emoticons displayed beside each paragraph: the first icon from the left allows to annotate the text with the label I feel worried by this; the second one with the label I don't understand this. By clicking on any of these emoticons, the corresponding text is highlighted either in pink or yellow (respectively) and associated with either labels. Patients can select any portion of the IC text, even across multiple paragraphs, and associate it with one of these two "emotions" (called reactions), i.e. worry and perplexity. Patients are told that by associating a reaction with any part of the IC, they are indirectly requesting assistance by a member of the caring staff. In particular, submitting an IC with reactions makes the system send a message either to a doctor, in case of doubts associated with the 'I don't understand this' label, or to a nurse, in case of the 'I feel worried' reaction. The IC cannot be approved and officially "signed", until all of the inserted reactions have been withdrawn by the patient, after receiving due assistance and support.

The system also automatically highlights the words of the IC that it recognises to be associated with a specific definition in a glossary of medical terms previously stored in the system files. When the user clicks on one of these hyperlinked words, the corresponding definition is displayed in a small word balloon (see Figure 2, where the definition of the term 'Terapia' - Therapy - is displayed once the user has clicked on the corresponding hyperlink).

\section{ThE QUANTITATIVE USER STUDY}

The DICE application was interactively designed at our lab, under our supervision, and the last version was developed by one of the authors. To test the application, we involved 24 prospective users in a series of short user tests, in which the application was evaluated in terms of efficiency (i.e. time to completion) and effectiveness (i.e. error rate) in controlled experimental conditions. After each session, we administered a closed-ended questionnaire. In what follows we report the results of this user study.

The usability study encompassed the administration of a usability questionnaire to evaluate the User Experience of the latest version of the prototype. This questionnaire encompassed 2 parts: the first part was aimed at measuring user 


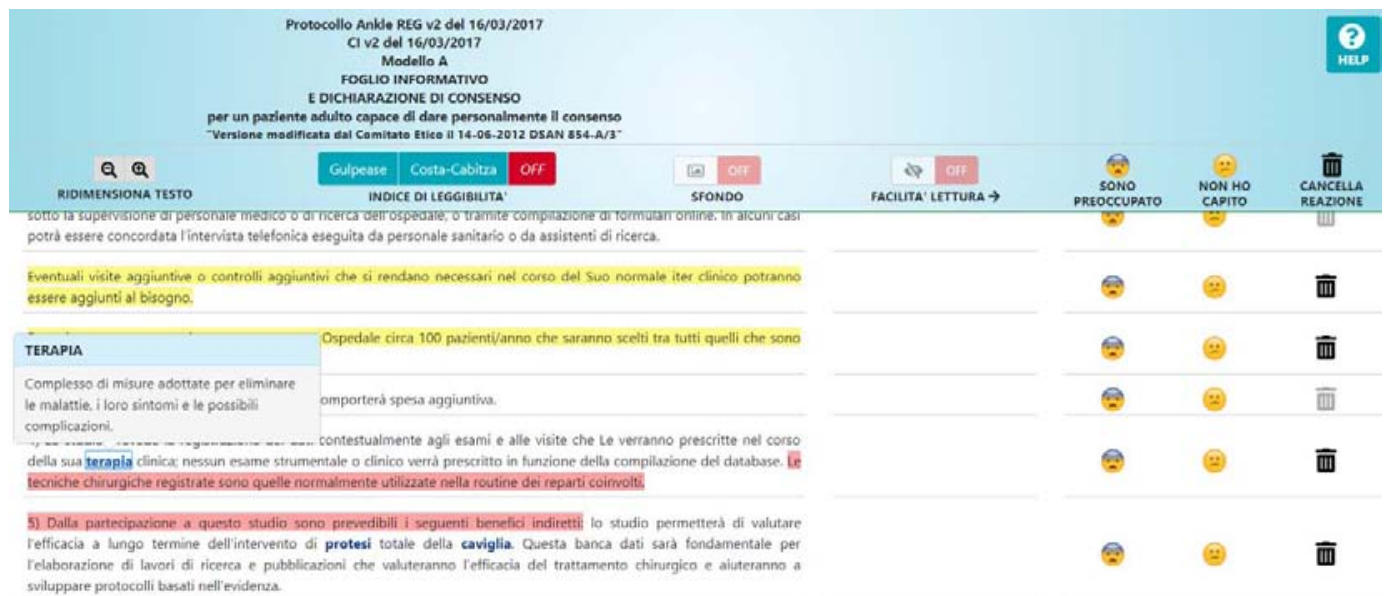

Figure 2. Screenshot of the main screen of the application.

satisfaction and was based on the UEQ questionnaire ${ }^{3}$ [23]; the second part was based on two items we conceived ad-hoc about the use of the prototype. The questionnaire was administered to 24 users: this sample was built on a convenience basis: in particular, $42 \%$ of the sample were women, and $50 \%$ of respondents were younger than 25 ; the great majority ( $75 \%$ of the population) of respondents had a high school diploma, with a medium to high familiarity for Web application. The participants filled in the questionnaire after using application for approximately 20-25 minutes for the direct involvement in the user test hinted at above.

1) The questionnaire UEQ: The User Experience Questionnaire (UEQ) contains 6 scales with 26 semantic differential items [23]. We chose this questionnaire for its widespread use, the known simplicity of semantic differentials, the availability of a validated Italian translation, and for the availability of a usability benchmark, which was built aggregating the responses of almost 20,000 participants involved in more than 400 studies on software applications. The results reported in Figure 3 show that the application was appreciated especially in terms of novelty, efficiency and perspicuity, which means that the product is easy to get familiar with, and users can solve their tasks without unnecessary effort.

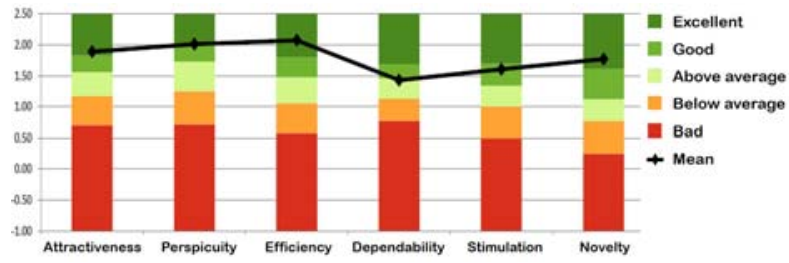

Figure 3. The DICE usability scores with respect to the UEQ benchmark, depicted as a bar chart.

\footnotetext{
${ }^{3}$ https://www.ueq-online.org/
}

Later, on a selected number of items, we applied hypothetical testing procedures to determine whether the respondents' responses showed any significant difference according to gender and Web familiarity. To this aim, we performed a Mann-Whitney $U$ test to assess whether there were significant differences between how attractive the system was perceived by male $(\mathrm{N}=14)$ and female respondents $(\mathrm{N}=$ 10) on a 7-value semantic differential ranging from very attractive to totally unattractive. The results are reported in Figure 4. According to this test, we found a statistically significant difference concerning the attractiveness of the system perceived by the men (Median $=2.5$ ) and women (Median $=2)(U=33, p=0.033)$. Since the lower the score, the more attractive the system was perceived, we found that men perceived the prototype as significantly less attractive than the female respondents. We can speculate that this significant difference may be due to the fact that males feel less comfortable in expressing emotions than females [24].

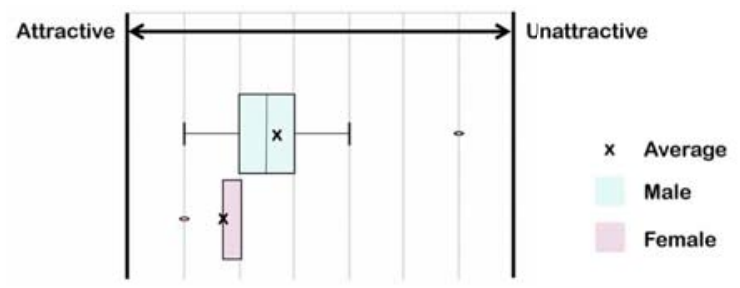

Figure 4. Boxplot for the responses given to the UEQ item "attractive unattractive" for female and male users.

We performed the same test on 20 participants to determine if there was any significant difference also in regard to intuitiveness (see Figure 5) between the respondents who claimed to have high familiarity with Web applications $(\mathrm{N}=$ 9) and those who claimed to have less than high familiarity $(\mathrm{N}=11)$. According to this test, we found a non significant difference between the median perceptions of intuitiveness 
by those with a high familiarity $($ Median $=2)$ and those with a medium-low familiarity (Median $=2, \mathrm{U}=30.5, \mathrm{p}=0.16$ ). This result suggests that the application was found equally intuitive by expert users and those who claimed to use Web applications seldom, and indeed these latter ones found the interface even (slightly) more intuitive.

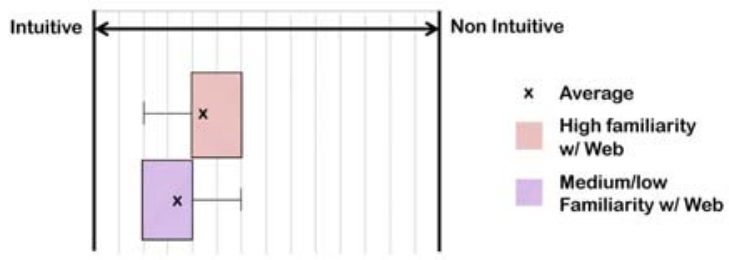

Figure 5. Boxplot for the item "intuitive - non intuitive" for respondents with different familiarity with Web-based applications (high vs. medium/low, self-assessed).

2) Ad-hoc Items: The 24 users involved in the user study had finally to answer two items that had been conceived to assess the degree of resistance to use, which we interpreted as the embarrassment of users to explicitly express emotions and feelings about the content of the IC through the system. The items were expressed in the following terms:

1) Expression of lack of understanding: On a scale from 1 (very little) to 6 (a lot) how embarrassing or uncomfortable would it be for you to express through the system that you didn't understand something that you read in the informed consent?

2) Expression of concern: On the same ordinal 6-value scale, how embarrassing or uncomfortable would it be for you to express through the system that something that you read in the informed consent worries you?

In Table I and Figure 6 we summarise the responses given by the respondents in terms of average response, its $95 \%$ confidence interval (ME stands for Margin of Error, i.e. half the CI interval), mode, median and rate of responses either above or below the middle option.

Table I

DESCRIPTIVE STATISTICS FOR THE RESPONSES TO THE AD-HOC ITEMS.

\begin{tabular}{l|cc} 
& Lack of understanding & Concern \\
\hline Mean & 2.88 & 2.42 \\
ME $_{@ 95 \%}$ & 0.43 & 0.47 \\
Mode & 2 & 2 \\
Median & 2.5 & 2 \\
Negative rate & $0.71(17)$ & $0.79(19)$ \\
Positive rate & $0.29(7)$ & $0.21(5)$ \\
ME $_{@ 95 \%}$ & 0.18 & 0.16
\end{tabular}

The responses collected show that no negative emotion prevails over the other. Moreover, we can also state that users would not feel excessively embarrassed or uncomfortable in expressing either lack of understanding nor concerns through the system. This finding results from performing a Zeta Score Test to compare the rate of responses indicating either low or high embarrassment to both items. Low embarrassment was associated with responses lower than or equal to 3 ; high embarrassment was associated with responses higher than or equal to 4 . Based on this test, we found significant differences in the rate of low and high embarrassment responses for both the lack of understanding item $(0.7 \pm 0.2$ vs. $0.3 \pm 0.2$, respectively; $\mathrm{z}$ score $=2.91, \mathrm{p}$ $=0.004)$ and the concern item $(0.79 \pm 0.16$ vs. $0.21 \pm 0.16$, $\mathrm{z}$ score $=4.02, \mathrm{p}<0.0001)$.

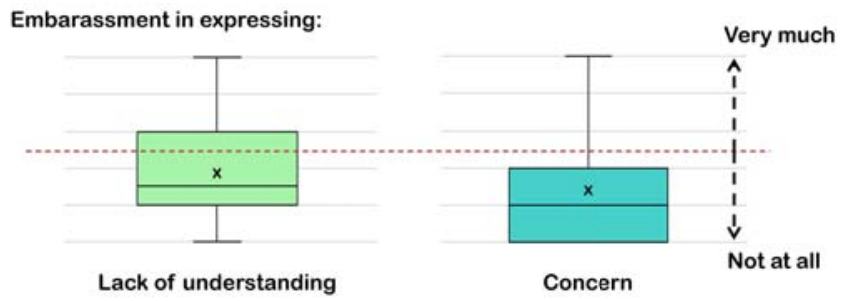

Figure 6. Boxplots representing the response distributions for the ad-hoc items; item 1 regards the embarrassment in expressing lack of understanding; item 2 the embarrassment in expressing concern.

\section{THE QUALITATIVE USER STUDY}

In order to get further insight on the real-world adoption of the DICE system by health professionals and patients, we also interviewed some key informants who could represent the main stakeholders involved in the IC process. To this aim, we involved 6 key-informants: 2 patients who had recently underwent surgery and had to consider the related IC thoroughly; 2 physicians with some experience in IC drafting, and 2 nurses, who deal with patients' worries and concerns on a daily basis. We conceived this part of the study as a semi-structured interview, to guarantee both flexibility and consistency in the questions asked to each respondent. Questions regarded the potential for DICE to improve the IC experience, and its main strengths and weaknesses in the light of the interviewee's direct experience. Each interview lasted approximately 35 minutes and was transcribed by one author. The analysis of the transcriptions allowed us to detect the following main issues.

First of all, the professional participants (nurses and physicians) expressed a concern regarding the lack of time in hospital work; also the patients noticed that relatively little time was spent on reading and signing the informed consent. Physicians said that the amount of time they usually have to prepare the patient for the surgery includes the formal approval of the consent and that this latter usually takes no more than 5 minutes: in their facility, doctors usually summarise the content of the IC verbally to the patients indicating the parts that the patients should consider more carefully, and patients sign it in a short while. Both the physicians involved strongly agreed about the fact that an application as DICE would require strong modifications in 
the routine and pre-surgery workflow. One stated that "the timing, the approach and the mindset about the consent process should be changed from head to foot". While either of them denied that creating some room and additional time for this process would be good, both the physicians and the nurses believed this could be nice but also impossible to have: "At the moment there is no time. I agree that it is far from ideal, but this is what we have!", said one physician. However, one nurse was less pessimist about that: "in most cases the surgeon could find some little more time for the consent before the surgery starts, obviously if it is the patient who asks for due clarifications about the procedure she is about to undertake".

Secondly, another problem that was raised by the interviewees regards the necessary skills that the patients should have to use such an application like DICE. One nurse said that "a lot of people here aren't so familiar with electronic devices. Many of our patients are elderly, and therefore for them reading an informed consent on a multi-touch tablet could be an even more frustrating or anxious experience than reading it on paper.".

Another problem identified by all the participants was related to the use of graphometric signature or, more generally, to the legal obligation of having the IC be signed on paper. The participants agreed upon the fact that DICE could be used by patients to read the consent and possibly call for the care giver support even if at the end of the process they would have to put their signature on the paper-based document. This could result in a cumbersome procedure (where the patient should trust the nurses that the electronic and paper-based ICs are identical in content), and additional waste of time.

On the other hand, the participants also emphasised some benefits of using such a system in a hospital ward. The first one regarded the functionality of having the system display the word balloons with the definition of the most difficult medical terms in the IC text. Both patients admitted to have read a few difficult words, whose meaning they did not know and, above all, that they were ashamed to ask what those words were about exactly. In particular, one patient said "I like [this functionality] because physicians love to use many specific terms that are not so easy to understand or for which you don't understand what they mean with respect to your condition, but asking for any explanations is difficult because one doesn't want to seem ignorant". For the similar reason, the patients were both positively intrigued by the possibility to express the reaction "I dont understand". They told us to believe that making this kind of request mediated by a computer system could make it easier to express doubts and require more explanations when they are due. To this latter regard, however, one of the nurses expressed a concern that giving the patients the capability to say "I am worried" for each paragraph of the IC could make them more anxious, not less, and load the nurses of an additional explicit responsibility and hence of the burden to reassure the patients more often than with the traditional paper-based IC.

\section{CONCLUSIONS}

In this study we have reported about the user study that we performed to assess the usability and real-world adoptability of an application of electronic informed consent, called Digital Informed Consent (DICE), which we designed and developed to encourage patients take an active attitude with respect to the content of the IC and ask for support if needed.

We reported of the findings from a computer-assisted online interview with 701 patients of a large teaching hospital in Italy, which show the importance to give patients tools by which all of their questions and doubts, about the procedures they have to undergo, could be duly and timely addressed: approximately one patient out of eight admitted that not all of their information needs were satisfied before surgery and that, therefore, the consent was not completely "informed".

Then, we reported about a standard questionnaire-based usability study that involved 24 representative users who were previously involved in some user tests (not reported in this study). The results of this study show that the prototype was appreciated for a number of usability dimensions (see Figure 3), especially novelty, and perspicuity, that is the extent the system is easy to learn. This was also confirmed by a hypothesis testing procedure, which did not find any significant difference in the intuitiveness perceived by expert and non-expert users. Interestingly, we also observed a statistically significant difference in how male and female users liked the system (cf. the attractiveness item in the UEQ questionnaire), with men who expressed a significant minor appreciation. We also found that users would not generally feel embarrassed or uncomfortable in expressing through the system that they have not understood something about the informed consent or that something about it worries them. This suggests that patients could perceive as valuable the functionality by which an eIC allows users to indicate passages that need further explanations or emotional support, and use the application in practice.

However, the good indications that we could draw from the questionnaire-based study were only marginally confirmed by a more qualitative study in which we interviewed six potential stakeholders of an application like DICE. In particular, the patients appreciated the capability to get an explanation of the most difficult medical terms at their fingertips. Although the capability to annotate the text in terms of their emotional reactions was not disliked by the patients, the care givers involved observed how this kind of functionality could make the actual adoption of the tool difficult in current workflows and hospital practices in light of the chronic lack of time and health operators who could timely address the right demands that such a tool could bring to light. In general, an electronic informed consent 
that is aimed at creating more (not fewer) opportunities of interaction between patients and care givers was seen as practically incompatible with their agenda, and to require additional time that cannot be expected and planned in the current hospital workflow.

For this reason, this study can be considered a contribution in the HCI debate about the hiatus between empiricallygrounded, and yet ideal, assessments of user experience, and the need to design useful applications for real and situated practices [25], where multiple perspectives and interests can clash and hinder innovation, even if it is conceived and proposed with the best of intentions.

\section{REFERENCES}

[1] D. J. Mazur, "Court viewpoints and medical decision making," $M D M$, vol. 6, no. 4, pp. 224-230, 1986.

[2] A. Paris, C. Brandt, C. Cornu, P. Maison, C. Thalamas, and J.-L. Cracowski, "Informed consent document improvement does not increase patients' comprehension in biomedical research," BJCP, vol. 69, no. 3, pp. 231-237, 2010.

[3] R. Barrett, "Quality of informed consent: measuring understanding among participants in oncology clinical trials." in Oncology nursing forum, vol. 32, no. 4, 2005.

[4] L. Stunkel, M. Benson, L. McLellan, N. Sinaii, G. Bedarida, E. Emanuel, and C. Grady, "Comprehension and informed consent: assessing the effect of a short consent form," IRB, vol. 32, no. 4, p. 1, 2010.

[5] G. S. De Oliveira Jr, J. L. Holl, R. J. McCarthy, Z. A. Butt, J. Nouriel, K. McCaffery, and M. S. Wolf, "Overestimation of mortality risk and preoperative anxiety in patients undergoing elective general surgery procedures: a propensity matched analysis," International Journal of Surgery, vol. 12, no. 12, pp. 1473-1477, 2014.

[6] A. Ali, D. Altun, B. H. Oguz, M. Ilhan, F. Demircan, and K. Koltka, "The effect of preoperative anxiety on postoperative analgesia and anesthesia recovery in patients undergoing laparascopic cholecystectomy," Journal of anesthesia, vol. 28, no. 2, pp. 222-227, 2014.

[7] J. B. Williams, K. P. Alexander, J.-F. Morin, Y. Langlois, N. Noiseux, L. P. Perrault, K. Smolderen, S. V. Arnold, M. J. Eisenberg, L. Pilote et al., "Preoperative anxiety as a predictor of mortality and major morbidity in patients aged $>$ 70 years undergoing cardiac surgery," The American journal of cardiology, vol. 111, no. 1, pp. 137-142, 2013.

[8] A. J. Gogos, R. B. Clark, M. M. Bismark, R. L. Gruen, and D. M. Studdert, "When informed consent goes poorly: a descriptive study of medical negligence claims and patient complaints," MJA, vol. 195, no. 6, pp. 340-344, 2011.

[9] S. Tariq and J. Woodman, "Using mixed methods in health research," JRSM short reports, vol. 4, no. 6, p. 2042533313479197, 2013.

[10] P. Shenoy, "Electronic informed consenting: A boon to modernize consenting process," Perspectives in clinical research, vol. 6, no. 4, p. 173, 2015.

[11] M. C. Rowbotham, J. Astin, K. Greene, and S. R. Cummings, "Interactive informed consent: randomized comparison with paper consents," PloS one, vol. 8, no. 3, p. e58603, 2013.
[12] T. Krishnamurti and N. Argo, "A patient-centered approach to informed consent: results from a survey and randomized trial," MDM, vol. 36, no. 6, pp. 726-740, 2016.

[13] B. W. Palmer, N. M. Lanouette, and D. V. Jeste, "Effectiveness of multimedia aids to enhance comprehension of research consent information: a systematic review," IRBEthics and Human Research, vol. 34, no. 6, p. 1, 2012.

[14] M. M. Issa, E. Setzer, C. Charaf, A. L. Webb, R. Derico, I. J. Kimberl, and A. S. Fink, "Informed versus uninformed consent for prostate surgery: the value of electronic consents," The Journal of urology, vol. 176, no. 2, pp. 694-699, 2006.

[15] M.-A. Hwang and I. J. Kwak, "Description of a mobile-based electronic informed consent system development," Studies in health technology and informatics, vol. 216, p. 897, 2015.

[16] L. McNair and A. Costello, "Electronic informed consent: A new industry standard," 2014.

[17] W. Rowan, Y. OConnor, L. Lynch, and C. Heavin, "Exploring the situational approach to decision making: User econsent on a health social network," World Academy of Science, Engineering and Technology, vol. 11, no. 11, pp. 2487-2492, 2017.

[18] J. Wilbanks, "Design issues in e-consent," The Journal of Law, Medicine \& Ethics, vol. 46, no. 1, pp. 110-118, 2018.

[19] J. Sugarman, D. C. McCrory, D. Powell, A. Krasny, B. Adams, E. Ball, and C. Cassell, "Empirical research on informed consent: an annotated bibliography," Hastings Center Report, vol. 29, no. 1, pp. s1-s42, 1999.

[20] D. W. Fitzgerald, C. Marotte, R. I. Verdier, W. D. Johnson Jr, and J. W. Pape, "Comprehension during informed consent in a less-developed country," The Lancet, vol. 360, no. 9342, pp. 1301-1302, 2002.

[21] M. K. Paasche-Orlow, H. A. Taylor, and F. L. Brancati, "Readability standards for informed-consent forms as compared with actual readability," NEJM, vol. 348, no. 8, pp. 721-726, 2003

[22] J. Flory and E. Emanuel, "Interventions to improve research participants' understanding in informed consent for research: a systematic review," Jama, vol. 292, no. 13, pp. 1593-1601, 2004.

[23] M. Schrepp, A. Hinderks, and J. Thomaschewski, "Construction of a benchmark for the user experience questionnaire (ueq)." IJIMAI, vol. 4, no. 4, pp. 40-44, 2017.

[24] T. B. Kashdan, A. Mishra, W. E. Breen, and J. J. Froh, "Gender differences in gratitude: Examining appraisals, narratives, the willingness to express emotions, and changes in psychological needs," Journal of personality, vol. 77, no. 3, pp. 691-730, 2009.

[25] S. Greenberg and B. Buxton, "Usability evaluation considered harmful (some of the time)," in Proceedings of the SIGCHI conference on Human factors in computing systems. ACM, 2008, pp. 111-120. 